Pacific Journal of Mathematic 


\section{AUTOMORPHIC GROUP REPRESENTATIONS}

\section{ROBERT J. BLATTNER}

1. Introduction In this paper we investigate certain representations of groups as * -automorphisms of rings of operators. More particularly, we are interested in finding conditions on the group, representation, and ring which guarantee the production of outer automorphisms of the ring. The exhibition of outer automorphisms has been considered before, notably by Singer in a paper [9] which intensively analyses the automorphism group of one of the finite factors constructed by von Neumann. Although we also shall be concerned with finite rings, our results do not overlap Singer's.

Segal, in [8], introduced the notion of skew distribution over a real Hilbert space $\mathfrak{S}$. He singled out one in particular, the Clifford distribution over $\mathfrak{g}$, which admits a representation $\Gamma$ of the orthogonal group $\mathscr{O}(\mathfrak{S})$ of $\mathfrak{S}$ into the automorphism group of the ring $\mathfrak{A}$ associated to the distribution. Section 2 of the present paper gives (mostly without proofs) a variant of Segal's construction of $\mathfrak{A}$ and $\Gamma$, which is more suitable for our calculations. Section 3 states and begins the proof of Theorem 1, which completely classifies (vis à vis innerness) the automorphisms of $\mathfrak{U}$ arising from $\Gamma$. The proof is completed in $\S 4$ and 5 .

In $\S 6$, we introduce the notion of a continuous automorphic group representation and show that any locally compact group satisfying the second axiom of countability may be represented as outer automorphisms of the Clifford distribution ring. Finally, Theorem 2 shows that any continuous automorphic representation of an open simple Lie group on a finite ring is essentially outer.

We shall make free use of the standard theory of operators and rings of operators as found in [6] and [3]. For the theory of measurable operators and gage spaces see [7].

The author would like to thank I. E. Segal for bringing the problems solved in this paper to his attention.

2. Preliminaries. Let $\mathfrak{T}$ be a real Hilbert space, $\mathfrak{I}$ the tensor algebra over $\mathfrak{T}, \mathfrak{I}$ the ideal generated by elements of the form $x \otimes x-(x, x) 1$. Set $\mathfrak{E}=\mathfrak{I} / \mathfrak{\Im}$, the Clifford algebra over $\mathfrak{S}$ with respect to the quadratic form $x \rightarrow(x, x)$, and $*=$ the main anti-automorphism of $\mathbb{E}=$ the antiautomorphism of $\mathbb{C}$ arising from the anti-automorphism of $\mathfrak{T}$ which sends

$$
x_{1} \otimes \cdots \otimes x_{n} \rightarrow x_{n} \otimes \cdots \otimes x_{1} .
$$

Received April 10, 1958. 
As usual, we will consider $\mathfrak{S}$ as embedded in $\mathfrak{S}$.

Any central linear functional $\theta$ on $\sqrt{5}$ has the property that $\theta\left(x_{1} \cdots x_{n}\right)=0$ whenever $\left\{x_{1}, \cdots, x_{n}\right\}$ are an orthogonal set in $\mathfrak{E}$. Since those elements, together with 1 , span $\mathfrak{E}$, there is (up to a multiplicative factor) at most one such $\theta$ on $\mathfrak{c}$. Let us produce one. Following Chevalley [2], we let $\mathfrak{F}=$ the exterior algebra over $\mathfrak{S}$, multiplication indicated by $\wedge$, and $\mathscr{C}=$ the algebra of endomorphisms of $\mathfrak{F}$. $\mathfrak{S}$ is considered embedded in $\mathfrak{F}$. For each $x \in \mathfrak{F}$, let $\delta_{x}$ be the unique antiderivation of $\left(\xi\right.$ such that $\delta_{x} y=(x, y) 1$ for all $y \in \mathfrak{S}$, and let $l_{x}$ be the operator of left multiplication by $x$ in $\left(5\right.$. The mapping $x \rightarrow l_{x}+\delta_{x}$ of $\mathfrak{S}$ into $\mathscr{C}$ extends to a homomorphism $\Psi$ of $\mathbb{E}$ into $\mathscr{C}$. We let $\tau(u)=\Psi(u) 1$ for $u \in \mathbb{E}$. It is easy to show that $\tau$ is a one-to-one linear map of $\mathbb{5}$ onto 5 .

Now the inner product $(\cdot, \cdot)$ on $\mathfrak{S}$ extends to a real Hilbert inner product, also called $(\cdot, \cdot)$, on $₹$. We set $\theta(u)=(\tau(u), 1)$. It is clear that $\theta$ is linear and that $\theta(1)=1$. We shall show that $\theta\left(v^{*} u\right)=(\tau(u), \tau(v))$. This will establish the centrality of $\theta$ and will also show that $(u, v)=$ $\theta\left(v^{*} u\right)$ is a Hilbert inner product on $(5$ making $\tau$ into an isometry. It suffices to prove the above when $u=x_{1} \cdots x_{n} y_{1} \cdots y_{r}$ and $v=x_{1} \cdots x_{n}$ $z_{1} \cdots z_{s}$ where $\left\{x_{1}, \cdots, x_{n}, y_{1}, \cdots, y_{r}, z_{1}, \cdots, z_{s}\right\}$ (n,r,s possibly 0 ) form an orthonormal set in $\mathfrak{S}$, since the $u \otimes v$ for all such pairs $u, v$ span $\sqrt[5]{ } \otimes(5 . \quad B u t$

$$
\begin{aligned}
& \theta\left(v^{*} u\right)=\theta\left(z_{s} \cdots z_{1} x_{n} \cdots x_{1} x_{1} \cdots x_{n} y_{1} \cdots y_{r}\right)=\theta\left(z_{s} \cdots z_{1} y_{1} \cdots y_{r}\right) \\
& \left.\quad=z_{s} \wedge \cdots \wedge z_{1} \wedge y_{1} \wedge \cdots \wedge y_{r}, 1\right)=1 \text { or } 0
\end{aligned}
$$

according as $r=s=0$ or not. Thus

$$
\begin{aligned}
& \theta\left(v^{*} v\right)=\left(x_{1} \wedge \cdots \wedge x_{n} \wedge y_{1} \wedge \cdots \wedge y_{r}, x_{1} \wedge \cdots \wedge x_{n} \wedge z_{1} \wedge \cdots \wedge z_{s}\right) \\
& \quad=(\tau(u), \tau(v))
\end{aligned}
$$

as desired.

Let $\mathfrak{D}$ be the complexification of $\mathbb{C}$ and extend the inner product on $\sqrt{5}$ in the usual way to a (complex) Hilbert inner product on $\mathfrak{D}$. Let $\Re$ be the completion of $\mathbb{D}$. * may be extended by conjugate linearity and closure to be a conjugation on $\Omega$. We note that if $\left\{e_{i}\right\}$ is an orthonormal basis for $\mathfrak{E}$, then $\left\{e_{i_{1}} e_{i_{2}} \cdots e_{i_{r}}\right\}\left(i_{1}<i_{2}<\cdots<i_{r} ; r=0,1, \cdots\right)$ is an orthonormal base for $\Re$, where the indices $i$ have been linearly ordered in some fashion. We shall adopt the notation $e_{A}, A$ a finite set of indices, to mean $e_{i_{1}} e_{i_{2}} \cdots e_{i_{r}}$ where $i_{1}<\cdots<i_{r}$ and $A=\left\{i_{1}, \cdots, i_{r}\right\}$. Conventionally $e_{\phi}=1$.

For any element $u \in \mathfrak{D}$, let $L_{u}^{\prime \prime}$ be the operator with domain $\mathfrak{D}$ defined by $L_{u}^{\prime \prime} a=u a, a \in \mathfrak{D}$. It is easily seen that $L_{x}^{\prime \prime}, x a$ unit vector of $\mathfrak{T}$, is an isometry of $\mathfrak{D}$ onto $\mathfrak{D}$. Since $\mathfrak{D}$ is spanned algebraically by products 
of the form $x_{1} \cdots x_{n}, x_{i}$ unit vectors in $\mathfrak{g}$, we conclude that $L_{u}^{\prime \prime}, u \in \mathfrak{D}$, is a continuous operator on the normed linear space $\mathfrak{D}$. Thus $\left(\Re, \mathfrak{D},{ }^{*}\right)$ forms a Hilbert algebra, the left ring $\mathfrak{A}$ of which is a factor of type II, when $\mathfrak{S}$ is infinite dimensional, which is the only case we shall consider [8]. Let $\mathfrak{B}$ be the algebra of all bounded elements of $\left(\Re, \mathfrak{D},{ }^{*}\right)$. $L_{a}$ and $R_{a}, a \in \mathfrak{B}$, will denote the closure of the left and right multiplication operators respectively by $a$ on the domain $\mathfrak{B}$. The maps $a \rightarrow L_{a}$ and $a \rightarrow R_{a}$ are an isomorphism and anti-isomorphism of $\mathfrak{B}$ onto $\mathfrak{A}$ and $\mathfrak{U}^{\prime}$ respectively. For $x \in \Re$, we define $L_{x}^{\prime}$ as the operator with domain $\mathfrak{B}$ such that $L_{x}^{\prime} a=R_{a} x, a \in \mathfrak{B}$. Then $L_{x}$ is defined to be $\left(L_{x^{*}}^{\prime}\right)^{*}$, the notation agreeing with the above when $x \in \mathfrak{B} . \quad L_{x}$ is always measurable with respect to $\mathfrak{A}[7]$.

3. The representation $\Gamma$. Any orthogonal transformation $U$ on $\mathfrak{S}$ extends canonically to an automorphism of $\mathfrak{I}$ which leaves $\mathfrak{\Im}$ invariant and thus induces an automorphism of $\sqrt{5}$, which we denote $\Gamma(U) . \quad \Gamma(U)$ is defined by

$$
\Gamma(U)\left(x_{1} \cdots x_{n}\right)=\left(U x_{1} \cdots U x_{n}\right) \text { for } x_{1}, \cdots, x_{n} \in \mathfrak{S} .
$$

Clearly $\Gamma(U)$ commutes with *. The functional $\theta \circ \Gamma(U)$ is again a central linear functional on $\sqrt{5}$ and $\theta(\Gamma(U) 1)=\theta(1)=1$. Hence $\theta \circ \Gamma(U)=\theta$ so that $\Gamma(U)$ is an isometry on $\Subset$. The automorphism $\Gamma(U)$ then extends to an automorphism of $\left(\Re, \mathfrak{D},{ }^{*}\right)$ which leaves $\mathfrak{D}$ invariant. $\Gamma$ is clearly a faithful representation of the orthogonal group of $\mathfrak{S}$ into the automorphism group of $(\Re, \mathfrak{D}, *)$. $\Omega$ will denote the automorphism $\Gamma(-I)$. $\Omega^{2}=I$, so that $\Omega$ is the direct sum of two subspaces $\Omega^{+}$and $\Omega^{-}$defined by $\Omega x=x$ or $-x$ according as $x \in \Omega^{+}$or $\Omega^{-}$.

$\Gamma(U)$ is an inner automorphism if there exists a unitary element $u \in \mathfrak{B}$ such that $\Gamma(U)=L_{u} R_{u^{*}}$. Since $\mathfrak{A}$ is a factor, $u$ is determined up to a multiplicative constant of modulus 1 by $\Gamma(U)$. Now

$$
\begin{aligned}
& \Gamma(U)=\Gamma((-I) U(-I))=\Omega L_{u} R_{u *} \Omega=\left(\Omega L_{u} \Omega\right)\left(\Omega R_{u^{*}} \Omega\right) \\
& \quad=L_{\Omega u} R_{\Omega u^{*}}=L_{\Omega u} R_{(\Omega u)^{*}} .
\end{aligned}
$$

Hence $\Omega u=\lambda u, \lambda$ a constant. Clearly $\lambda= \pm 1$ so that either $u \in \Omega^{+}$or $u \in \mathfrak{R}^{-}$. In the former case $\Gamma(U)$ is called even, in the latter, odd. Those inner automorphisms $\Gamma(U)$ which are even form a subgroup of the group of all inner automorphisms of the type $\Gamma(U)$.

In order to classify the automorphisms $\Gamma(U)$ according to the above categories, we introduce the following notation: Let $\mathscr{G}^{+}$be the set of orthogonal transformations $U$ on $\mathfrak{S}$ such that $I-U$ is of Hilbert-Schmidt class and whose eigenspace belonging to -1 has even dimension; let $\mathscr{G}^{-}$contain all those $U$ such that $I+U$ is Hilbert-Schmidt and whose eigenspace belonging to +1 has odd dimension. Set $\mathscr{C}_{0}=\mathscr{G}^{+} \cup \mathscr{G}^{-}$. 
THEOREM 1. $\Gamma(U)$ is inner if and only if $U \in \mathscr{G}_{0}$. If $\Gamma(U)$ is inner, it is even if and only if $U \in \mathscr{C}^{+}$.

Let $\left\{e_{i}\right\}_{i \in J}$ be an orthonormal basis of $\mathfrak{S}_{\mathfrak{Z}}$, where $J$ is a totally ordered index set, and let $U$ be a fixed orthogonal transformation. Set $f_{i}=U e_{i}$ and $V_{i}=L_{f_{i}} R_{e_{i}}$.

LEMma 1. The subspace \& of vectors left invariant by all the $V_{i}$ has dimension $=0$ or 1 . If $\operatorname{dim} \mathfrak{Q}=0, \Gamma(U)$ is outer ; if $\operatorname{dim} \mathfrak{Q}=1$, \& contains a unitary element $u \in \mathfrak{B}$ such that $\Gamma(U)=L_{u} R_{u^{*}}$.

Proof. Suppose $\Gamma(U)=L_{u} R_{u^{*}}, u$ a unitary in $\mathfrak{B}$. Then $\Gamma(U) e_{i}=$ $L_{u} R_{u^{*}} e_{i}$ or $f_{i}=U e_{i}=u e_{i} u^{*}$, all $i$. Thus $f_{i} u e_{i}=u$ or $V_{i} u=u$, all $i$. Therefore $\operatorname{dim} \mathfrak{Q} \geqq 1$ if $\Gamma(U)$ is inner.

Next suppose $\operatorname{dim} \mathfrak{Z} \geqq 1$ and let $0 \neq x \in \mathfrak{L}$. Then $R_{f_{i}} x^{*}=L_{e_{i}} x^{*}$ so that $L_{L_{e_{i}} x^{*}}^{\prime}=L_{R_{f_{i}} x^{*}}^{\prime}$. For any element $a \in \mathfrak{B}, L_{L_{e_{i} x^{*}} a}^{\prime} a=R_{a} L_{e_{i}} x^{*}=L_{e_{i}} R_{a} x^{*}$ $=L_{e_{i}} L_{x^{*}}^{\prime} a$ so that $L_{L_{e_{i}} x^{*}}^{\prime}=L_{e_{i}} L_{x^{*}}^{\prime}$. Similarly $L_{R_{f_{i}} x^{*}}^{\prime}=L_{x^{*}}^{\prime} L_{f_{i}}^{\prime}$. Taking adjoints, we have

$$
\left(L_{L_{e_{i}} x^{*}}^{\prime}\right)^{*} \supseteqq\left(L_{x^{*}}^{\prime}\right)^{*} L_{e_{i}}{ }^{*}=L_{x} L_{e_{i}} \text { and }\left(L_{R_{f_{i}} x^{*}}^{\prime}\right)^{*} \supseteqq\left(L_{f_{i}}^{\prime}\right)^{*}\left(L_{x^{*}}^{\prime}\right)^{*}=L_{f_{i}} L_{x} \text {. }
$$

Therefore, using - to indicate strong product [7], $L_{x} \cdot L_{e_{i}}=L_{f_{i}} \cdot L_{x}$. Again taking adjoints, $L_{e_{i}} \cdot L_{x}^{*}=L_{x}^{*} \cdot L_{f_{i}}$. This implies $L_{f_{i}} \cdot L_{x} \cdot L_{x}^{*}=$ $L_{x} \cdot L_{x}^{*} \cdot L_{f_{i}}$; that is, the positive measurable operator $L_{x} L_{x}^{*}$ commutes with each $L_{f_{i}}$. Thus every spectral projection of $L_{x} L_{x}^{*}$ commutes with each $L_{f_{i}}$. But the $\left\{L_{f_{i}}\right\}$ are a self-adjoint set of generators for $\mathfrak{A}$ and $\mathfrak{A}$ is a factor. Therefore each spectral projection of $L_{x} L_{x}^{*}$ is either 0 or $I$, whence $L_{x} L_{x}^{*}=\lambda I, \lambda$ a positive constant. A similar argument shows that $L_{x}^{*} L_{x}=\lambda I$. Thus we have shown that $L_{x}$ is bounded and that $\lambda^{-1 / 2} L_{x}$ is unitary so that $\lambda^{-1 / 2} x$ is a unitary element in $\mathfrak{B} \cap \mathbb{R}$.

Let $u=\lambda^{-1 / 2} x$. Then $f_{i} u e_{i}=u$, and hence $u e_{i} u^{*}=f_{i}$. Therefore the automorphisms $\Gamma(U)$ and $L_{u} R_{u^{*}}$ agree on the $\left\{e_{i}\right\}$ which are a set of generators for $\left(\Re, \mathfrak{D},{ }^{*}\right)$; that is, $\Gamma(U)=L_{u} R_{u^{*}}$. Suppose now that $0 \neq y \in \mathbb{R}$ also. Then $y \in \mathfrak{B}, y y^{*}=y^{*} y=\mu 1, \mu$ a positive constant. $v=$ $\mu^{-1 / 2} y$ is also a unitary $\in \mathfrak{B} \cap \mathbb{R}$ and $\Gamma(U)=L_{v} R_{v^{*}}$. But this implies that $v=\zeta u, \zeta$ a constant; that is, $y=\mu^{1 / 2} \zeta u$. Hence $\operatorname{dim} \mathbb{Q}=1$.

DEFINITION 1. For any orthogonal transformation $U$ on $\mathfrak{S}$, the subspace $\mathcal{Q}$ of $\Omega$ is called its characteristic subspace.

It is clear from Lemma 1 that the characteristic subspace depends only on $U$ and not on the choice of a basis $\left\{e_{i}\right\}$ for $\mathfrak{S}$.

4. The determinant condition. In this section we will show that if $I^{\prime}(U)$ is an even inner automorphism, then $I-U$ is Hilbert-Schmidt. 
This will be achieved in a series of lemmas. We adhere to the notation of $\S 2$.

Lemma 2. Let $U$ be an orthogonal transformation on $\mathfrak{g}$ and let $P$ be the projection on its characteristic subspace. Then

$$
\lim _{A} \operatorname{det}_{k, l \in A}\left(2^{-1}(I+U) e_{k}, e_{l}\right)=(P 1,1) .
$$

(Here "lim" means limit according to the set of finite subsets $A$ of $J$, directed upward by inclusion. The determinant is expanded with respect to the total order on the elements of $A$.)

Proof. We first introduce some notation: $f_{A}$ will denote $\Gamma(U) e_{A}$; $P_{i}=$ the projection on the invariant subspace of $V_{i}$ (see Lemma 1 ); if $A=\left\{i_{1}, \cdots, i_{r}\right\}\left(i_{1}<\cdots<i_{r}\right)$ then $P_{A}=P_{i_{1}} \cdots P_{i_{r}}$.

Since the $V_{i}$ mutually commute, the $P_{A}$ are projections which mutally commute. Clearly $P=$ strong- $\lim _{A} P_{A}$. In addition, $V_{i}^{2}=I$, all $i$. This imples that $P_{i}=2^{-1}\left(I+V_{i}\right)$; that is, $P_{i} a=2^{-1}\left(a+f_{i} a e_{i}\right)$ for $a \in \mathfrak{B}$. Iterating this, we calculate that

$$
P_{A} 1=2^{-r} \sum_{B \subseteq} f_{A} e_{B}^{*}
$$

where $r$ is the cardinality of $A$. Hence $\left(P_{A} 1,1\right)=2^{-r} \sum_{B C_{A}}\left(e_{B}, f_{B}\right)$.

Fix $B \subseteq A$ and suppose $B=\left\{j_{1}, \cdots, j_{s}\right\}\left(j_{1}<\cdots<j_{s}\right)$. Then

$$
\begin{gathered}
\left(e_{B}, f_{B}\right)=\left(\tau\left(e_{B}\right), \tau\left(f_{B}\right)\right)(\operatorname{see} \S 2)=\left(e_{j_{1}} \wedge \cdots \wedge e_{j_{s}}, f_{i_{1}} \wedge \cdots f_{j_{s}}\right) \\
\quad=\operatorname{det}_{k, l \in B}\left(e_{k}, f_{l}\right)=\operatorname{det}_{k, l \in B}\left(e_{k}, U e_{l}\right)=\operatorname{det}_{k, l \in B}\left(U e_{k}, e_{l}\right) .
\end{gathered}
$$

Hence

$$
\left(P_{A} 1,1\right)=2^{-r} \sum_{B \subseteq A} \operatorname{det}_{k, l \in B}\left(U e_{k}, \varrho_{l}\right),
$$

which we recognize to be

$$
\operatorname{det}_{k, l \in A}\left(2^{-1}(I+U) e_{k}, \varepsilon_{l}\right) .
$$

Passing to the limit on $A$, we have the lemma.

Note that the lemma shows that $\lim _{A} \operatorname{det}_{k, l \in A}\left(2^{-1}(I+U) e_{k}, e_{l}\right)$ depends only on $U$ and not on the particular choice of basis. Hence we may write $\operatorname{det}\left(2^{-1}(I+U)\right)$ without fear of confusion. This motivates the following.

Definition 2. An operator $T$ on $\mathfrak{g}$ will be said to have a determinant if, for every choice of an orthonormal basis $\left\{e_{i}\right\}_{i \in J}$ ( $J$ totally ordered), $\lim _{A} \operatorname{det}_{k, l \in A}\left(T e_{k}, e_{l}\right)$ exists and is independent of the choice of basis. We write $\operatorname{det}(T)$ for the common limit. (Cf. the treatment in [5].) 
To make use of the conclusion of Lemma 2, we must prove a short preliminary result. For any operator $T, \sigma(T)$ will denote the spectrum of $T$.

Lemma 3. Let $S$ be a self-adjoint operator on $\mathfrak{S}$ and let $\left\{S_{\alpha}\right\}$ be a net of self-adjoint operators and $\left\{Q_{x}\right\}$ a net of projections (same index set) such that:

(1) $S=$ strong- $\lim _{\alpha} S_{\alpha}$;

(2) $S_{\alpha}=Q_{\alpha} S_{\alpha} Q_{\alpha}$ and $I=$ strong- $\lim _{\alpha} Q_{\alpha}$. Then $\sigma(S) \subseteq$ topological lim $\inf _{\alpha} \sigma\left(S_{\alpha} \mid Q_{\alpha} \mathfrak{S}\right)$.

Proof. Let $\lambda \in \sigma(S)$ and $\varepsilon>0$ be given. Since $S$ is self-adjoint, we can find a unit vector $x \in \mathfrak{W}$ such that $\|(S-\lambda I) x\|<\varepsilon / 4$. We may then find an index $\alpha_{0}$ such that $\alpha>\alpha_{0}$ implies $\left\|\left(S_{\alpha}-S\right) x\right\|<\varepsilon / 4$ and $\left\|Q_{\alpha} x\right\| \geqq 1 / 2$. Then $\left\|\left(S_{\alpha}-\lambda I\right) x\right\|<\varepsilon / 2$, whence $\left\|\left(S_{\alpha}-\lambda Q_{\alpha}\right)\left(Q_{\alpha} x\right)\right\|<\varepsilon / 2$. Set $y_{\alpha}=$ $Q_{a} x /\left\|Q_{a} x\right\|$. Then $y_{a}$ is a unit vector in $Q_{a} \mathfrak{K}$. We have shown that $\left\|\left(S_{\alpha}-\lambda Q_{\alpha}\right) y_{\alpha}\right\|<\varepsilon$. This implies that $\sigma\left(S_{\alpha} \mid Q_{\alpha} \mathfrak{S}\right)$ contains a point within $\varepsilon$ of $\lambda, \alpha>\alpha_{0}$.

We shall apply this to the situation in the following lemma.

LEMmA 4. Let the operator $T$ on $\mathfrak{S}$ have a determinant $\operatorname{det}(T)=c \neq 0$ and suppose $\|T\| \leqq 1$. Then $I-T^{*} T$ is of trace class.

Proof. Chose a basis $\left\{e_{i}\right\}_{i \in J}, J$ totally ordered. We shall take as our index set the set of all finite subsets $A$ of $J . Q_{A}=$ the projection on the subspace of $\mathfrak{S}_{\mathcal{E}}$ spanned by the $e_{i}, i \in A$. Clearly $Q_{A} \rightarrow I$ strongly. Set $S=T^{*} T, S_{A}=Q_{A} T^{*} Q_{A} T Q_{A}$, and $T_{A}=Q_{A} T Q_{A} / Q_{A} \mathcal{S}_{\text {. }}$. Then $S_{A} \rightarrow S$ strongly and $S_{A} / Q_{A} \mathfrak{S}=T_{A}^{*} T_{A}$. Lemma 3 asserts that $\sigma\left(T^{*} T\right) \leqq$ topological $\lim \inf _{A} \sigma\left(T_{A}^{*} T_{A}\right)$.

Now $\operatorname{det}\left(T_{A}^{*} T_{A}\right)=\left(\operatorname{det} T_{A}\right)^{2}$ so that the hypotheses of the lemma assert that $\lim _{A} \operatorname{det}\left(T_{A}^{*} T_{A}\right)=c^{2} \neq 0$ and $\left\|T^{*} T\right\| \leqq 1$, implying each $\left\|T_{A}^{*} T_{A}\right\| \leqq 1$. Clearly $\sigma\left(T^{*} T\right)$ and $\sigma\left(T_{A}^{*} T_{A}\right) \subseteq[0,1]$. Given $\varepsilon<1$, let $N(A, \varepsilon)=$ cardinality of $\sigma\left(T_{A}^{*} T_{A}\right) \cap[0, \varepsilon)$ and $N(\varepsilon)=$ cardinality of $\sigma\left(T^{*} T\right) \cap[0, \varepsilon)$. Choose a set $A_{0}$ such that $\operatorname{det}\left(T_{A}^{*} T_{A}\right) \geqq 2^{-1} c^{2}$ for $A \supseteqq A_{0}$. Since $\operatorname{det}\left(T_{A}^{*} T_{A}\right)$ is the product of the eigenvalues of $T_{A}^{*} T_{A}$, we must have that $N(A, \varepsilon) \leqq$ $\log 2^{-1} c^{2} / \log \varepsilon$ for $A \supseteqq A_{0}$. Therefore $N(\varepsilon) \leqq \log 2^{-1} c^{2} / \log \varepsilon$. This shows that $\sigma\left(T^{*} T\right)$ is pure point spectrum except possibly for the value 1 and that $\sigma\left(T^{*} T\right)$ has only 1 as a cluster point.

$\mathfrak{S}$ is the direct sum of the eigenspaces of $T^{*} T$. Choose a new basis, again called $\left\{\boldsymbol{e}_{i}\right\}_{i \in J}$, adapted to this direct decomposition of $\mathfrak{S}$. We use the notation of the previous paragraphs (with respect to the new basis). Each $e_{i}$ is an eigenvector belonging to an eigenvalue $\lambda_{i}$ of $T^{*} T$. For every finite subset $A$ of $J$ we have 


$$
\prod_{i \in A} \lambda_{i}=\operatorname{det}\left(T^{*} T \mid Q_{A} \mathfrak{W}\right)
$$

But

$$
T^{*} T\left|Q_{A} \mathfrak{W}-T_{A}^{*} T_{A}=Q_{A} T^{*}\left(I-Q_{A}\right) T Q_{A}\right| Q_{A} \mathfrak{Q},
$$

a positive operator on $Q_{A} \mathfrak{S}$. Now the determinant of the sum of two positive operators on a finite dimensional Hilbert space is greater than the determinant of either operator. Hence

$$
\prod_{i \in A} \lambda_{i} \geqq \operatorname{det}\left(T_{A}^{*} T_{A}\right) \text {. }
$$

As $A \uparrow, \prod_{i \in A} \lambda_{i} \downarrow$ since $0 \leqq \lambda_{i} \leqq 1$ for all $i \in J$. It follows that $\Pi_{i} \lambda_{i}$ exists. Moreover, $\Pi_{i} \lambda_{i} \geqq c^{2}>0$ since $\lim _{A} \operatorname{det}\left(T_{A}^{*} T_{A}\right)=c^{2}$. Therefore $\Pi_{i} \lambda_{i}$ converges absolutely, so that $\sum_{i}\left(1-\lambda_{i}\right)<\infty$; that is, $I-T^{*} T$ is of trace class.

Lemma 5. Let $\Gamma(U) L_{u} R_{u^{*}}, u$ a unitary operator of $\mathfrak{B} \cap \Re^{+}$. Then $I-U$ is Hilbert-Schmidt.

Proof. Fix a basis $\left\{e_{i}\right\}_{i \in J}, J$ totally ordered. Each $e_{i}$ is a selfadjoint unitary element of $\mathfrak{B}$. Hence for any finite subset $A$ of $J, e_{A}$ (notation as in $\S 2$ ) is a unitary in $\mathfrak{B}$. For each $e_{i}$ we define $U_{e_{i}}$ to be that orthogonal transformation on $\mathfrak{S}$ which leaves $e_{i}$ invariant and multiplies the other elements of the basis by -1 . It is easy to see that $\Gamma\left(U_{e_{i}}\right)=L_{e_{i}} R_{e_{i}}$. In general, we define $U_{e_{A}}$ to be $U_{e_{i_{1}}} \cdots U_{e_{i_{r}}}$ where $A=\left\{i_{1}, \cdots, i_{r}\right\}\left(i_{1}<\cdots<i_{r}\right)$. Then $\Gamma\left(U_{e_{A}}\right)=L_{e_{A}} R_{e_{A}}$; and $U_{e_{A}} \in \mathscr{C}^{+}$or $\mathscr{C}^{-}$and $\Gamma\left(U_{e_{A}}\right)$ is even or odd according as $A$ has even or odd cardinality. It is clear that all the $U_{e_{A}}$ are self-adjoint.

Let $\Gamma(U)=L_{u} R_{u^{*}}, u$ a unitary element of $\mathfrak{B} \cap \Re^{+}$. Then $u=\sum_{A} \lambda_{A} e_{A}$, the summation being extended over the $A$ of even cardinality. Pick a $\lambda_{B} \neq 0$. Then $u=e_{B}\left(\sum_{A} \lambda_{A}^{\prime} e_{A}\right) ; \lambda_{A}^{\prime}$ 's different constants, Setting $v=$ $\sum_{A} \lambda_{A}^{\prime} e_{A}, v$ is a unitary in $\mathfrak{B} \cap \Omega^{+}$such that $(v, 1)=\lambda_{\phi}^{\prime}=\lambda_{B} \neq 0$. Set $V=U_{e_{B}} U$. Then

$$
\Gamma(V)=\Gamma\left(U_{e_{B}}\right) \Gamma(U)=\left(L_{e_{B}{ }^{*}} R_{e_{B}}\right)\left(L_{u} R_{u^{*}}\right)=L_{e_{B}{ }^{*} u} R_{\left(e_{B}{ }^{*} u\right)^{*}}=L_{v} R_{v^{*}} .
$$

Since $U_{e_{B}} \in \mathscr{G}^{+}, I-U$ will be Hilbert-Schmidt if and only if $I-V$ is. Thus we may assume without loss of generality that $(u, 1) \neq 0$.

If $P$ is the projection on the characteristic subspace of $U$, our assumption implies that $(P 1,1) \neq 0$. Setting $T=2^{-1}(I+U)$, we conclude from Lemmas 2 and 4 that $I-T^{*} T$ is of trace class. This says that

$$
I-\frac{1}{4}\left(I+U^{*}\right)(I+U)=\frac{1}{4}\left(2 I-U-U^{*}\right)
$$


is of trace class. Hence $2 I-U-U^{*}=(I-U)^{*}(I-U)$ is of trace class so that $I-U$ is Hilbert-Schmidt.

\section{Completion of proof.}

Lemma 6. If $U \in \mathscr{G}^{+}\left[\right.$respectively $\left.\mathscr{G}^{-}\right]$, then $\Gamma(U)$ is an even [odd] inner automorphism.

Proof. We use the notation of Lemma 5. Let $U \in \mathscr{G}^{+}$[respectively $\left.\mathscr{C}^{-}\right]$. Then the eigenspace $\mathfrak{M}$ of $U$ belonging to the eigenvalue $-1[+1]$ is of even [odd] dimension. Let $\left\{e_{i}\right\}_{i \in A}$ be a basis for $\mathfrak{M}$. Then $U_{e_{A}} \in \mathscr{C}^{+}$ $\left[\mathscr{S}^{-}\right]$. Set $V=U_{e_{A}} U$. It is easily seen that $V \in \mathscr{O}^{+}$and that the eigenspace belonging to -1 has dimension 0 . If the lemma can be proved for $V$, it will follow for $U$ since $U=U_{e_{A}} V$ will then be the product of an even [odd] and an even inner automorphism. Thus we may assume without loss of generality that $U \in \mathscr{G}^{+}$and that $U$ has no eigenvectors belonging to -1 .

$\mathfrak{S}$ is the direct sum of the eigenspaces of $(I-U)^{*}(I-U)$. These spaces are all finite dimensional except possibly that belonging to 0 . These subspaces all reduce $U$ and on the 0 -eigenspace $U=I$. Using the classical reduction of an orthogonal transformation on a Euclidean space and remembering that $U$ has no eigenvectors belonging to -1 , we see that $\mathfrak{S}$ is the direct sum of a countable number of 2-dimensional subspaces $\mathfrak{K}_{n}(n=1,2, \cdots)$ and a subspace $\mathfrak{W}_{0}$, each of which reduces $U$, and such that $U$ is irreducible on every $\mathfrak{S}_{n}$ and $U=I$ on $\mathfrak{S}_{0}$. Let $\left\{e_{i}\right\}_{i \in J}$ be a basis for $\mathfrak{g}$ adapted to this direct decomposition. With respect to this basis

$$
U \mid \mathfrak{S}_{n}=\left(\begin{array}{cc}
\cos \theta_{n} & -\sin \theta_{n} \\
\sin \theta_{n} & \cos \theta_{n}
\end{array}\right),
$$

(where the basis elements of $\mathfrak{S}$ have been suitably ordered). We readily calculate $\operatorname{det}\left(2^{-1}(I+U)\right)$ to be

$$
\Pi_{n} \operatorname{det}\left(\frac{1}{2}(I+U) \mid \mathfrak{S}_{n}\right)=\prod_{n}\left(\frac{1+\cos \theta_{n}}{2}\right) .
$$

For any operator $T$, we denote its Hilbert-Schmidt norm by $\|T\|_{2}$. Then

$\|I-U\|_{2}^{2}=\sum_{n}\left\|(I-U) \mid \mathfrak{S}_{n}\right\|_{2}^{2}=4 \sum_{n}\left(1-\cos \theta_{n}\right)=8 \sum_{n}\left(1-\frac{1+\cos \theta_{n}}{2}\right)$.

Hence

$$
\prod_{n}\left(\frac{1+\cos \theta_{n}}{2}\right)
$$


converges absolutely. By Lemma 2 , this says that $(P 1,1) \neq 0$, where $P$ is the projection on the characteristic subspace of $U$. Thus $P \neq 0$ and $\Gamma(U)$ is inner by Lemma 1 . Finally, $\Gamma(U)$ must be even. In fact, suppose $\Gamma(U)=L_{u} R_{u^{*}}, u$ unitary in $\mathfrak{B} \cap \Omega^{-}$. Since $P$ is the projection on the subspace generated by $u$ and since $1 \in \mathfrak{K}^{+}$, we conclude $P 1=0$, a contradiction.

Lemma 7. If $\Gamma(U)$ is inner, then $U \in \mathbb{S}_{0}$.

Proof. Suppose $I(U)$ is odd. Let $e_{1}$ be a unit vector in $\mathfrak{S}$. Then $\Gamma\left(U_{e_{1}}\right)$ (notation as in Lemma 5) is odd. It follows that $\Gamma\left(U_{e_{1}} U\right)$ is even, so that $I-U_{e_{1}} U$ is Hilbert-Schmidt. Since $I+U_{e_{1}}$ is Hilbert-Schmidt, so is $I+U=\left(I+U_{e_{1}}\right)-U_{e_{1}}\left(I-U_{e_{1}} U\right)$.

Let now $\Gamma(U)$ be even [odd]. We know that $I-U$ [respectively $I+U]$ is Hilbert-Schmidt. Suppose that the eigenspace of $U$ belonging to $-1[+1]$ is of odd [even] dimension. Then $-U \in \mathbb{S}^{-[\text {respectively }}$ $\left.\mathscr{G}^{+}\right]$so that $\Gamma(-U)$ is also inner by Lemma 6 . Therefore $\Omega=\lambda \Gamma\left(U^{*}(-U)\right)$ is inner. We shall be through if we can show that $\Omega$ is outer.

Suppose then that $\Omega$ is inner. Since $I-(-I)$ is not Hilbert-Schmidt, Lemma 5 implies that $\Omega$ must be odd. Let $\Omega=L_{u} R_{u^{*}}, u$ unitary in $\mathfrak{B} \cap \Re^{-}$. Picking a basis $\left\{e_{i}\right\}_{i \in J}, J$ totally ordered, for $\mathfrak{S}, u=\sum \lambda_{A} e_{A}$, the summation being extended over all finite $A \subseteq J$ of odd cardinality.

We let $\&$ be the characteristic subspace of $-I$ and adopt the notation of Lemmas 1 and 2. We have $P_{i} u=u$ for each $i \in J$. Now

$$
P_{i} u=\sum_{A} \lambda_{A} \cdot \frac{1}{2}\left(e_{A}-e_{i} e_{A} e_{i}\right) .
$$

Since $A$ has odd cardinality, $e_{i} e_{A} e_{i}=e_{A}$ or $-e_{A}$ according as $i \in A$ or $i \notin A$. Hence $\lambda_{A}=0$ unless $i \notin A$ for all $i$; that is, all $\lambda_{A}=0$, which is ridiculous. This concludes the proof of Lemma 7 and, with it, Theorem 1.

6. Automorphic representations of topological groups. The mapping $\Gamma$ is a representation of the orthogonal group $\mathcal{C}(\mathfrak{S})$ of $\mathfrak{g}$ as automorphisms of the Hilbert algebra $(\mathfrak{S}, \mathrm{D}, *)$. Every automorphism of the Hilbert algebra gives rise to an automorphism of its left ring $\mathfrak{A}$ via the isomorphism $b \leftrightarrow L_{b}$ of the bounded algebra $\mathfrak{B}$ and $\mathfrak{A}$. Conversely, every $*$-automorphism of $\mathfrak{A}$ gives rise to one of the Hilbert algebra (by the uniqueness of the normalized central trace on $\mathfrak{B}$ ) and the correspondance is univalent. Henceforth we identify these two types of automorphisms.

Let $\mathscr{A}$ be the ${ }^{*}$-automorphism group of $\mathfrak{U}$. If $\alpha \in \mathscr{A}, T \in \mathfrak{A}, T^{\infty}$ denotes the image of $T$ by $\alpha$. We have then

$$
L_{b}^{\Gamma(u)}=L_{\Gamma(u) b}=\Gamma(U) L_{b} \Gamma(U)^{*}
$$


for $U \in \mathscr{O}(\mathfrak{S})$ and $b \in \mathfrak{B}$. Now it is easy to check that the maps $U \rightarrow \Gamma(U) b, b \in \mathfrak{D}$, are continuous in the norm of $\Re$ when $\mathcal{O}(\mathfrak{S})$ is given the strong operator topology (as henceforth it shall be). Hence $\Gamma$ is continuous from $\mathscr{O}(\mathfrak{S})$ to the unitaries of $\Re$ in the strong topology. It follows that, for each $T \in \mathfrak{A}$, the map

$$
U \rightarrow T^{\Gamma(u)}=\Gamma(U) T \Gamma(U)^{*}
$$

is continuous from $\mathcal{O}(\mathfrak{S})$ to $\mathfrak{U}$ in the strong, and hence the weak topology. $\Gamma$ is thus a continuous automorphic representation, in the following sense.

DEFINITION 3. Let $\mathfrak{A}$ be a ring of operators, $\mathscr{A}$ its * -automorphism group, $G$ a topological group. A representation $\rho$ of the abstract group $G$ into $\mathscr{A}$ is called a continuous automorphic representation on $\mathfrak{A}$ if, for every $T \in \mathfrak{A}$, the map $g \rightarrow T^{(\rho)}$ of $G$ into $\mathfrak{A}$ (in the weak topology) is continuous.

This continuity restriction is the weakest that can reasonably be imposed on $\rho$ and is independent of the particular spacial representation of $\mathfrak{A}$. We note that if $\rho$ is continuous in the above sense, then $g \rightarrow T^{\rho(g)}$ is strongly continuous. In fact, let $g_{\alpha} \rightarrow g$. Then

$$
\begin{gathered}
\left(T^{\rho\left(g_{\alpha}\right)}-T^{\rho(g)}\right)^{*}\left(T^{\rho\left(g_{\alpha}\right)}-T^{\rho(g)}\right) \\
=\left(T^{*} T\right)^{\rho\left(\theta_{\alpha}\right)}-\left(T^{*}\right)^{\rho\left(g_{\alpha}\right)} T^{\rho(g)}-\left(T^{*}\right)^{\rho\left(g_{\alpha}\right)} T^{\rho(g)}+\left(T^{*} T\right)^{\rho(g)} \rightarrow 0 \text { weakly. }
\end{gathered}
$$

Let now $G$ be a topological group, $\tau$ a continuous representation of $G$ into $\mathscr{O}(\mathfrak{L}), \mathfrak{A}$ the left ring of our Hilbert algebra, and $\mathscr{A}$ its automorphism group. Any $\alpha \in \mathscr{A}$ which leaves $\mathfrak{S}$ invariant will be called special. $\rho=\Gamma \circ \tau$ is then a continuous representation of $G$ as special automorphisms. Conversely, let $\rho$ be a special continuous automorphic representation on $\mathfrak{A}$. Then $\rho=\Gamma \circ \tau$, where $\tau$ is a representation of the abstract group $G$ into $\mathscr{O}(\mathfrak{S})$ (merely restrict $\rho$ to $\mathfrak{W}$ ). Let $a, b \in \mathfrak{W}$. Then $g \rightarrow(\tau(g) a, b)=\left(L_{a}^{\rho(g)} 1, b\right)$ is continuous so that $\tau$ is continuous.

With this in mind, we see that Theorem 1 has the following easy consequence.

COROLlaRY. Let $G$ be a locally compact group satisfying the second axiom of countability. Then $G$ has a special continuous automorphic representation $\rho$ such that if $\rho(g)$ is inner, then $g=e$.

Proof. Let $\tau_{1}$ be a faithful continuous representation of $G$ into some orthogonal group $\mathcal{O}\left(\mathfrak{S}_{1}\right), \mathfrak{S}_{1}$ a real Hilbert space of countable dimension; e.g., the left regular representation of $G$ (real functions). Let $\widehat{W}$ be the cardinality of a basis for $\mathfrak{g}$. Then $\mathfrak{S}$ is the $\widehat{s}$-fold direct copy of $\mathfrak{S}_{1}$ so that the direct sum of $\aleph^{N}$ copies of $\tau_{1}$, call it $\tau$, can be 
taken as a representatation of $G$ into $\mathscr{O}(\mathfrak{S})$. Since $\boldsymbol{H}$ is infinite, it is clear that $\tau(g) \varepsilon \mathscr{C}_{0}$ if and only if $\tau(g)=I$; that is, $g=e$. Set $\rho=\Gamma \circ \tau$.

It can also be deduced from Theorem 1 that for any special continuous automorphic representation $\rho$ of an open simple Lie group on $\mathfrak{A}$, $\rho^{-1}$ (inner automorphisms) is central. But this will follow from the more general statement in Theorem 2.

THEOREM 2. Let $G$ be an open simple Lie group, $\mathfrak{A}$ a finite ring of operators, and $\rho$ a non-trivial continuous automorphic representation of $G$ on $\mathfrak{A}$. Then $\rho(g)$ is inner only if $g$ is central.

The essential step is Lemma 8 below. It, together with an extension of a result of Kadison and Singer [4] to continuous projective unitary representation (for definitions, see below), will imply our theorem. Let $\mathfrak{A}$ be any ring of operators, $\mathscr{A}$ its automorphism group, $\mathscr{A}_{0}$ the subgroup of inner automorphisms, and $\mathscr{Z}$ the group of unitary operators in $\mathfrak{A}$. The map $\pi: \mathscr{U} \rightarrow \mathscr{A}_{0}$ defined by $T^{\pi(U)}=U T U^{*}, U \in \mathscr{U}, T \in \mathfrak{A}$, is a homomorphism onto $\mathscr{C}$ o whose kernel is the center $\mathscr{C}$ of $\mathscr{\mathscr { C }}$. If $\mathscr{U}$ is given the strong operator topology, then $\pi$ is a continuous automorphic representation. We set $\mathscr{P}=\mathscr{Q} / \mathscr{C}$ with the topology induced from $\mathscr{U}$ and, with some abuse of language, call $\mathscr{P}$ the projective unitary group of $\mathfrak{A}$; any continuous group representation into $\mathscr{P}$ will be called a continuous projective unitary representation on $\mathfrak{A} . \pi$ clearly induces a continuous isomorphism $\bar{\pi}$ of $\mathscr{P}$ onto $\mathscr{A}_{0}$.

Let $\tau$ be a continuous projective unitary representation of the topological group $G$ on $\mathfrak{A}$. Then $\rho=\bar{\pi} \circ \tau$ is an inner continuous automorphic representation of $G$ on $\mathfrak{A}$. Lemma 8 gives a partial converse.

LEMMA 8. Let $G$ be a locally compact group satisfying the second axiom of countability and let $\mathfrak{A}$ be a ring of operators on a separable Hilbert space $\mathfrak{W}$. Let $\rho$ be an inner continuous automorphic representation of $G$ on $\mathfrak{A}$. Then $\rho$ is induced by a continuous projective unitary repretation on $\mathfrak{A}$.

Proof. Let $\mathfrak{A}_{1}$ be the unit sphere of $\mathfrak{A}$ in the weak topology. $\mathfrak{A}_{1}$ is a compact separable metric space (since $\mathfrak{L}$ is separable). $G$ is a complete separable metric space and bears its left Haar measure $\mu$. Let $\left\{T_{i}\right\}$ be a dense sequence in $\mathfrak{X}_{1}$, and let $\mathfrak{S}$ be the set of all $(g, U) \in G \times \mathfrak{A}_{1}$ such that $U$ is unitary and $U T_{i}=T_{i}^{\mathrm{\rho}(g)} U$ for all $i$. The argument of Lemma $2(b)$, section $6, \S 2$, Chapter II of [3] shows that $\subseteq$ is a Borel, and hence an analytic, subset of $G \times \mathfrak{N}_{1}$. Let $\zeta$ be the canonical projection of $G \times \mathfrak{A}_{1}$ on $G$. Since $\rho$ is inner, $\zeta(\mathfrak{S})=G$. By Appendix $V$ of [3], there exists a measurable map (in the sense of Bourbaki [1]) $g \rightarrow U_{g}$ of $G$ into $\mathfrak{A}_{1}$ such that $\left(g, U_{g}\right) \in \mathfrak{S}, g \in G$. 
Thus we have a measurable map $g \rightarrow U_{g}$ of $G$ into $\mathscr{U}$ in the weak, and hence strong, topology such that $T_{i}^{\mathrm{p}(g)}=U_{g} T_{i} U_{g}^{*}$, all $i$. Taking weak limits of $\left\{T_{i}\right\}$, we have $T^{\rho(g)}=U_{g} T U_{g}^{*}$ for all $\mathrm{T} \in \mathfrak{A}_{1}$, and hence in $\mathfrak{A}$. Let $\psi$ be the canonical projection of $\mathscr{U}$ on $\mathscr{P}$ and set $\tau(g)=\psi\left(U_{g}\right)$. We know that $g \rightarrow \rho(g)=\pi\left(U_{g}\right)=\bar{\pi}(\tau(g))$ is a representation. Since $\bar{\pi}$ is univalent, $\tau$ is a representation. Since $\psi$ is continuous, $\tau$ is measurable from $G$ into $\mathscr{P}$. Hence there exists a compact $K \subseteq G$ such that $\mu(K)>0$ and $\tau \mid K$ is continuous. Using the representation property of $\tau$ we see easily that $\tau \mid K K^{-1}$ is continuous. But $K K^{-1}$ is a neighborhood of $e$ in $G[10]$. Therefore $\tau$ is continuous, as desired.

Proof of Theorem 2. For any ring of operators, the group of inner automorphisms is invariant in the full group of automorphisms. Let $G$ be an open simple Lie group and $C$ its center. Since every proper abstract invariant subgroup of $G$ is contained in $C$, the theorem asserts the non-existence of non-trivial inner continuous automorphic representations of $G$ on finite rings. Suppose, on the contrary, that $\rho$ is such a representation on the finite ring $\mathfrak{A}$ on the Hilbert space $\mathfrak{S}$. We shall first show that $\mathfrak{S}$ may be assumed separable.

For eace $g \in G$ choose a unitary $U_{g} \in \mathfrak{A}$ such that $U_{g} T U_{g}^{*}=T^{\rho(g)}, T \in \mathfrak{A}$. Let $\left\{g_{i}\right\}$ be a dense sequence in $G$ and let $\mathfrak{B}_{\circ}$ be the ${ }^{*}$-algebra (with unit) generated by the $U_{g_{i}}$ over the complex rationals. $\mathfrak{B}_{\circ}$ is countable. Let $\mathfrak{B}$ be the weak closure of $\mathfrak{B}_{\circ}$, a finite ring of operators. Clearly each $\rho\left(g_{i}\right)$ leaves $\mathfrak{B}_{\circ}$, and hence $\mathfrak{B}$, setwise invariant. The continuity of $\rho$ then implies that $\rho(g)$ leaves $\mathfrak{B}$ setwise invariant, $g \in G$. Set $\sigma(g)=\rho(g) \mid \mathfrak{B}$. Then $\sigma$ is a continuous automorphic representation on $\mathfrak{B}$ and each $\sigma\left(g_{i}\right)$ is inner by construction. The argument of the last paragraph shows that $\sigma(g)$ is inner, $g \in G$.

We next assert that $U_{g_{j}}^{\sigma\left(g_{i}\right)} U_{g_{j}}{ }^{*} \neq I$ for some $i, j$. Otherwise $U_{g_{i}} U_{g_{j}}=$ $U_{g_{j}} U_{g_{i}}$, whence $\rho\left(g_{i} g_{j}\right)=\rho\left(g_{j} g_{i}\right)$, all $i, j$. Taking limits, we see that $\rho$ maps the commutator subgroup of $G$ (=G itself) into the identity, a contradiction. Let then $x \in \mathfrak{S}$ be a vector not invariant under all the $U_{g_{j}}^{\sigma\left(g_{i}\right)} U_{g_{j}}{ }^{*}$. Set $\Re$ equal to the closure of $\mathfrak{B} x$ and $P$ equal to the smallest central projection of $\mathfrak{B}$ such that $P x=x$. $\Re$ reduces every $T \in \mathfrak{B}$ and the homomorphism $T \rightarrow T \mid K$ of $\mathfrak{B}$ onto $\mathfrak{B} \mid \Re$ is faithful and onto on the direct summand $\mathfrak{B P}$ of $\mathfrak{B}$. Since each $\sigma(g)$ is inner, each leaves the center of $\mathfrak{B}$ elementwise invariant. Therefore $\sigma$ induces by restriction an automorphic representation $\bar{\sigma}$ of $G$ on $\mathfrak{B P}$ and hence, via the above isomorphism, on $\mathfrak{B} \mid \Re$. Clearly $\bar{\sigma}$ is continuous and inner. It is also non-trivial, since $x \in \Re$. Lastly, $\mathfrak{B}_{\circ} x$ is a countable dence subset of $\Re$.

We thus see, returning to the first paragraph of the proof, that we can assume $\mathfrak{S}$ separable. Lemma 8 then implies the existence of a non-trivial continuous representation of $G$ into the projective unitary group $\mathscr{P}=\mathscr{U} / \mathscr{C}$ of $\mathfrak{A}$. We follow now the methods of [4]. Since 
$\mathfrak{T}$ is separable, $\mathfrak{A}$ is countably decomposable and hence carries a faithful normal positive (finite) central trace $\omega$. The space $\mathfrak{A}$ is then a preHilbert space in the norm $\|T\|_{2}^{2}=\omega\left(T^{*} T\right)$ and the unitaries of $\mathfrak{U}$ form a topological group $\mathscr{U}_{2}$ in the metric $d(U, V)=\|U-V\|_{2}$. The identity map of $\mathscr{U}$ onto $\mathscr{U}$ is continuous, hence so is the identity map of $\mathscr{P}$ onto $\mathscr{P}_{2}=\mathscr{U}_{2} / \mathscr{C}$. Therefore $G$ has a non-trivial continuous representation into $\mathscr{F}_{2}$. The metric $g$ on $\mathscr{C}_{2}$ is both left and right invariant. Hence $\mathscr{P}$ has a metric similarly invariant. The Lemma in [4] shows that $G$ has arbitrarily small invariant neighborhoods of the identity, an impossibility. This contradiction proves Theorem 2 .

\section{REFERENCES}

1. N. Bourbaki, Intégration, Paris, 1952.

2. C. Chevalley, The algebraic theory of spinors, New York, 1954.

3. J. Dixmier, Les algèbres d'opérateurs dans l'espace hilberiien, Paris, 1957.

4. R. V. Kadison and I. M. Singer, Some remarks on representations of connected groups, Proc. Nat. Acad. Sci. U. S. A. , 38 (1952), 419-423.

5. F. Riesz, Équations linéaraires à une infinité d'inconnues, Paris, 1913.

6. F. Riesz and B. Sz-Nagy, Functional analysis, London, 1956.

7. I. E. Segal, A non-commutative extension of abstract integration, Ann. of Math., 57 (1953), 401-475.

8. I. E. Segal, Tensor algebras over Hilbert spaces II, Ann. of Math., 63 (1956), 160-175.

9. I. M. Singer, Automorphisms of finite factors, Amer. J. Math., 77 (1955), 117-133.

10. A. Weil, L'intégration dans les groups topologiques et ses applications, Paris, 1940.

The University of California at Los ANgeles 



\section{PACIFIC JOURNAL OF MATHEMATICS}

\section{EDITORS}

\section{David Gilbarg}

Stanford University

Stanford, California

\section{R. A. Beaumont}

University of Washington

Seattle 5, Washington

\author{
A. L. Whiteman
}

University of Southern California Los Angeles 7, California

E. G. Straus

University of California

Los Angeles 24, California

\section{ASSOCIATE EDITORS}

\author{
E. F. BECKENBACH \\ C. E. BURGESS \\ M. HALL \\ E. HEWITT
}
A. HORN
V. GANAPATHY IYER
R. D. JAMES
M. S. KNEBELMAN
L. NACHBIN
I. NIVEN
T. G. OSTROM
H. L. ROYDEN

M. M. SCHIFFER

G. SZEKERES

F. WOLF

K. YOSIDA

\section{SUPPORTING INSTITUTIONS}

\author{
UNIVERSITY OF BRITISH COLUMBIA \\ CALIFORNIA INSTITUTE OF TECHNOLOGY \\ UNIVERSITY OF CALIFORNIA \\ MONTANA STATE UNIVERSITY \\ UNIVERSITY OF NEVADA \\ OREGON STATE COLLEGE \\ UNIVERSITY OF OREGON \\ OSAKA UNIVERSITY \\ UNIVERSITY OF SOUTHERN CALIFORNIA
}

\author{
STANFORD UNIVERSITY \\ UNIVERSITY OF TOKYO \\ UNIVERSITY OF UTAH \\ WASHINGTON STATE COLLEGE \\ UNIVERSITY OF WASHINGTON \\ * * * * \\ AMERICAN MATHEMATICAL SOCIETY \\ CALIFORNIA RESEARCH CORPORATION \\ HUGHES AIRCRAFT COMPANY \\ THE RAMO-WOOLDRIDGE CORPORATION
}

Mathematical papers intended for publication in the Pacific Journal of Mathematics should be typewritten (double spaced), and the author should keep a complete copy. Manuscripts may be sent to any one of the four editors. All other communications to the editors should be addressed to the managing editor, E. G. Straus at the University of California, Los Angeles 24, California.

50 reprints per author of each article are furnished free of charge; additional copies may be obtained at cost in multiples of 50 .

The Pacific Journal of Mathematics is published quarterly, in March, June, September, and December. The price per volume (4 numbers) is $\$ 12.00$; single issues, $\$ 3.50$. Back numbers are available. Special price to individual faculty members of supporting institutions and to individual members of the American Mathematical Society: $\$ 4.00$ per volume; single issues, $\$ 1.25$.

Subscriptions, orders for back numbers, and changes of address should be sent to Pacific Journal of Mathematics, 2120 Oxford Street, Berkeley 4, California.

Printed at Kokusai Bunken Insatsusha (International Academic Printing Co., Ltd.), No. 6, 2-chome, Fujimi-cho, Chiyoda-ku, Tokyo, Japan.

PUBLISHED BY PACIFIC JOURNAL OF MATHEMATICS, A NON-PROFIT CORPORATION

The Supporting Institutions listed above contribute to the cost of publication of this Journal, but they are not owners or publishers and have no responsibility for its content or policies. 


\section{Pacific Journal of Mathematics}

\section{Vol. 8, No. $4 \quad$ June, 1958}

Richard Arens, The maximal ideals of certain functions algebras ........ 641

Glen Earl Baxter, An operator identity ........................... 649

Robert James Blattner, Automorphic group representations ........... 665

Steve Jerome Bryant, Isomorphism order for Abelian groups ............ 679

Charles W. Curtis, Modules whose annihilators are direct summands...... 685

Wilbur Eugene Deskins, On the radical of a group algebra ............ 693

Jacob Feldman, Equivalence and perpendicularity of Gaussian

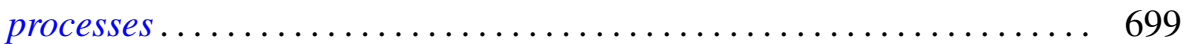

Marion K. Fort, Jr. and G. A. Hedlund, Minimal coverings of pairs by triples....................................... 709

I. S. Gál, On the theory of $(m, n)$-compact topological spaces ......... 721

David Gale and Oliver Gross, A note on polynomial and separable games........................................ 735

Frank Harary, On the number of bi-colored graphs ............... 743

Bruno Harris, Centralizers in Jordan algebras ................... 757

Martin Jurchescu, Modulus of a boundary component ............... 791

Hewitt Kenyon and A. P. Morse, Runs . . . . . . . . . . . . . . . . . . . . . . 811

Burnett C. Meyer and H. D. Sprinkle, Two nonseparable complete metric

spaces defined on $[0,1] \ldots \ldots \ldots \ldots \ldots \ldots \ldots \ldots \ldots \ldots \ldots . \ldots . \ldots . \ldots . \ldots 25$

M. S. Robertson, Cesàro partial sums of harmonic series expansions...... 829

John L. Selfridge and Ernst Gabor Straus, On the determination of numbers by their sums of a fixed order ........................ 847

Annette Sinclair, A general solution for a class of approximation

problems .................................

George Szekeres and Amnon Jakimovski, $(C, \infty)$ and $(H, \infty)$ methods of summation...................................... 867

Hale Trotter, Approximation of semi-groups of operators. ............. 887

L. E. Ward, A fixed point theorem for multi-valued functions ........... 921

Roy Edwin Wild, On the number of lattice points in $x^{t}+y^{t}=n^{t / 2} \ldots \ldots .929$ 